\title{
Unique pre-ß amyloid precursor protein (APP-A4) observations in ocular tissues from suspected abusive head trauma victims
}

Volume 7 Issue 3 - 2017 Keywords: ocular injury, abusive head trauma, inflicted head
injury

Abbreviations: AHT, abusive head trauma; BFHI, blunt force head injury; BS, brain swelling; COD, cause of death; DOD, date of death; DH, disc hemorrhage; EBS, endbulb swelling; GHII, global hypoxic-ischemic injury; IOP, intraocular pressure; LC, lamina cribrosa

\section{Introduction}

Abusive head trauma (AKA: Shaken Baby Syndrome, Shaken Impact Syndrome; Inflicted Head Injury; Whiplash Shake Syndrome, Non-Accidental Head Trauma) remains a major societal problem and is among the most controversial and complex medico-legal issues worldwide by virtue of the difficulty of achieving clear proof of criminal intent by suspected perpetrators. ${ }^{1,2}$ In the United States, trial by jury initiated by the local district attorney is the final arbiter. Trials typically involve testimonies from multiple witnesses including first responders, family members and medical experts for both the prosecution and defense.

Ocular injury, especially retinal hemorrhages, have been documented in $40-75 \%$ of reported AHT cases. ${ }^{3-5}$ Other features of fatal cases often include somnolence on admission to emergency rooms, subarachnoid and subdural hemorrhage, skull and other fractures, apnea and hypoxia related to severe intracranial pressure (ICP) elevation inhibiting blood flow to the brain resulting in severe brain edema with fontanelle widening. Upper cervical cord injury from exaggerated head movement including cervical cord compression during shaking has been suggested as the primary trigger for central nervous system (CNS) hypoxia. Intraocular and anterior optic nerve sheath hemorrhages, commonly observed, presumably result from basal cistern blood migrating forward under the influence of high intracranial pressure through the subdural and subarachnoid spaces around the nerve in continuity with the basal cistern.

Neuropathology examination at autopsy often demonstrates a necrotic "respirator brain" as well as multiple other features. ${ }^{6-8}$ Typical ocular pathology findings besides optic nerve sheath and retinal hemorrhages, sometimes include a macular ridge and hemorrhagic retinal schisis, and even retinal dehiscence. ${ }^{6}$ Papilledema in some is a likely a consequence of elevated intracranial pressure (ICP). Intraocular choroidal and retinal venous congestion secondary to high ICP inhibiting blood return to the cavernous sinus could contribute to retinal and vitreous hemorrhages and possibly to elevation of IOP, seldom if ever measured pre-mortem in AHT cases. Recent scanning electron microscopy (SEM) studies offer an explanation for the reportedly common macular hemorrhagic retinal schisis due to substantial vitreo-retinal attachments peculiar to infants. ${ }^{9}$

APP-A4 (AKA: Amyloid beta A4 protein, $\beta$ pre-amyloid human protein, Alzheimer precursor protein, Alzheimer protein,

\author{
Minckler DS, ${ }^{1,2}$ Charlson ES, ${ }^{1,2}$ Angele \\ Nalbandian ${ }^{1,2}$ \\ 'Departments of Laboratory Medicine and Ophthalmology, \\ University of California, USA \\ ${ }^{2}$ Department of Ophthalmology, University of California, USA
}

Correspondence: Don S Minckler, University of CaliforniaIrvine, Gavin Herbert Eye Institute 850 Health Sciences Road, Irvine, CA, USA, Tel 92697-4375, Fax (760) 840-1389, Email minckler@uci.edu

Received: December 15, 2016 | Published: February 13, 2017

neurofibrillary protein), came to high scientific interest in the late 1980s and is a widely available and sensitive neuropathology marker for identifying axonal injury consistent with severe shearing injury to myelinated and non-myelinated human CNS white matter. ${ }^{10-12}$ APP-A4, currently being investigated for its role in Alzheimer disease, is a type-I transmembrane protein with a complex multiple domain genetic organization, now recognized as an evolutionarily conserved protein participating in multiple essential intracellular processes in all vertebrates. The human APP gene is expressed in glial and neuronal cells, but also in almost all tissues that have been examined. ${ }^{13}$ Evidence suggests that a fragment of APP-A4 after $\alpha$-secretase cleavage displays neuroprotective and trophic properties and reduces the degeneration of dendrites in experimental injury. For example, post-traumatic administration of APP $\alpha$ reduced the number of apoptotic neurons in a mouse model of brain injury. ${ }^{14}$ Retinal ganglion cells (RGCs) manufacture the precursors in their cytoplasm and move them to sites of axonal injury in the rapid orthograde transport system, somewhat akin to first responders in civil disasters. ${ }^{11}$

\section{Materials and methods}

The selection of the first three cases was based on our observation of specific, not previously reported features, of optic nerve injury in AHT. The fourth case illustrates an important previously mentioned but not illustrated symmetry across companion eyes we found commonin our larger unpublished series. ${ }^{8}$ We do not have access to multiple sets of autopsy eyes from children expiring for non-suspected AHT as they are not routinely sent out for ophthalmic pathology examination. We believe demonstrating that symmetrically heavy staining by APP-A4 of retinal ganglion cells across companion eyes and dissimilar findings in companion optic nerves constitute adequate controls for our purposes and conclusions. Background staining in non-injured anterior optic nerve segments was always present, but tapered rapidly posterior to the lamina usually absent by $10 \mathrm{~mm}$ posterior to the globe.

An Appendix describes the APP-A4 technique. The cost per slide at our institution is $\$ 35.00 /$ each (Table 1). 
Table I Case Data, Modified from: Reichard RR, et al., ${ }^{8}$

\begin{tabular}{|c|c|c|c|c|c|c|c|c|c|}
\hline $\begin{array}{l}\text { \# Age } \\
\text { (mo) Sex }\end{array}$ & APP-A4 & DOD & $\mathbf{R H}$ & DH & ONH & SDH & BS & Sex & $\begin{array}{l}\text { Sex } \\
\text { Ddays }\end{array}$ \\
\hline & Findings of Interest & COD & & & POSH & SAH & BFHI & & \\
\hline & & MOD & & & & & GHII & & \\
\hline 1 & 40 years IHC & \#\#\#\#\#\#\# & $\mathrm{Fe}+\mathrm{OU}$ & Yes & No & Yes & Yes & No & 5 \\
\hline $10 \mathrm{mo}$ & stability APP-A4 +OU & $\mathrm{AHT}$ & RT OD & & No & Yes & Yes & & \\
\hline$M$ & & Suspect & \multicolumn{2}{|c|}{ RDS nasally near disc \& VH } & & & Yes & & \\
\hline 2 & APP-A4+ OU at & \#\#\#\#\#\#\# & OU; all & Yes OD & Yes & Yes & Yes & Yes & 2 \\
\hline $45 \mathrm{mo}$ & anterior edge of & Homicide at trial & layers & Fe neg. & Yes & Yes & Yes & & \\
\hline \multirow[t]{2}{*}{$\mathrm{F}$} & ON infarct OD & & Fe neg. & & OU & & Yes & & \\
\hline & & & & & Fe neg & & & & \\
\hline 3 & Contusion ON OD bracketed by & 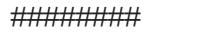 & OU all & No & Yes & Yes & Yes & Yes & 4 \\
\hline $4 \mathrm{mo}$ & +APP-A4 & $\mathrm{AHT}$ & layers & & Yes & Yes & Yes & & \\
\hline \multirow[t]{2}{*}{$\mathrm{F}$} & & Suspect & Mild VH & & FE neg & & Yes & & \\
\hline & & & Fe neg. & & & & & & \\
\hline $3 \mathrm{mo}$ & macular input \& EBS & Homicide at trial & Fe neg. & & No & No & Yes & & \\
\hline $\mathrm{F}$ & in lamina & & & & & & Yes & & \\
\hline
\end{tabular}

AHT:Abusive Head Trauma; BFHI: Blunt Force Head Injury; BS: Brain Swelling; COD: Cause of Death; DOD: Date of Death; DH: Disc Hemorrhage; EBS: Endbulb Swelling; GHII: Global Hypoxic-Ischemic Injury; IOP: Intraocular Pressure; LC: Lamina Cribrosa; AEB: Axonal End-Bulbs; MOD: Manner of Death; ONH: Optic Nerve Hemorrhage Within Parenchyma; APP: Amyloid Precursor Protein Immunostaining; Fe: Iron Staining; OR: Operating Room; PAS: Peripheral Anterior Synechiae; POSH: Perioptic Sheath Hemorrhage; RD: Retinal Detachment; RDS: Retinal Dehiscence; RH: Retinal Hemorrhage; po: Post Operatively; SAH: Intracranial Subarachnoid Hemorrhage; SDH: Intracranial Subdural Hemorrhage; SFx: Skull Fracture; RT: Retinal Tear; Sv: Survival Injury to Death in Days; VH: Vitreous Hemorrhage

\section{Results}

We obtained heavy APP-A4 staining in RGCs from the 40yearold Case \#1 demonstrating potential for remarkable stability and durability of the protein components in formalin-fixed paraffinized tissues (Figure 1). Obviously one such demonstration does not establish that any similarly agedor older paraffin material will reliably work with this marker. However this one example is a good reason to further explore the use of this APP-A4 marker in long-stored paraffin embedded human ocular tissue if results might be pertinent to ongoing human autopsy studies. Our larger series of cases will include several in which paraffin embedding dates back to 2010 , allowing better estimation of the marker's durability in general over at least 10years. Case \#2 demonstrated an optic nerve infarct beginning approximately $6 \mathrm{~mm}$ posterior to the globe, expanding to involve nearly the whole diameter of the nerve in deeper sections from the most posterior portion, some $29 \mathrm{~mm}$ behind the globe likely into the chiasm since the orbital and optic canal portions of the nerve together average approximately $29 \mathrm{~mm}$ in length (Figure 2). In this example, APP-A4 intra-axonal accumulations were found clearly outlining the anterior end of the infarct in agreement with routine Hematoxylin and Eosin (H\&E) and Periodic Acid-Schiff(PAS) stains (Figure 2A-D). While not surprising this does illustrate how blocked orthograde axonal transport is sharply interrupted by an adjacent infarction. Case \#3 illustrates for the first time both orthograde and retrograde axonal transport block corresponding to anterior and posterior sides of a hemorrhagic optic nerve contusion (Figure 3A-C \& Figure 4). Case \#4, to our knowledge is the best demonstration ever illustrated demonstrating bilaterally symmetrical APP-A4 macular input and classic end-bulb swellings in the lamina cribrosa OU, in our view reinforcing no need for nonAHT controls (Figure 5) ${ }^{8,9}$ The symmetry of APP-A4 staining of RGCs and optic nerve heads with laminar end-bulb swellings in axons across companion eyes in this case well document expected vigorous bilateral pre-mortem orthograde axonal transport physiology at least to the lamina cribrosa.

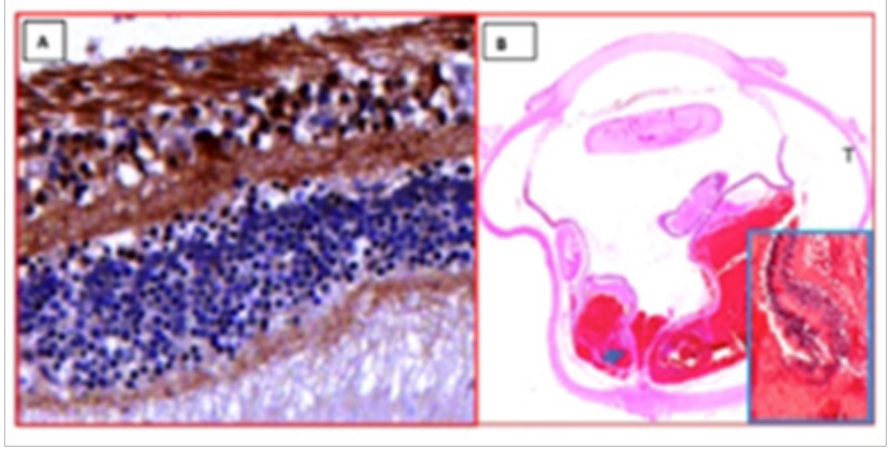

Figure I (A) Photomicrographs from Case \#I OD, IOP unknown, illustrating positive APP-A4+ macular retinal ganglion cells (RGCs) between nerve fiber layer and inner plexiform layer (APP-A4Hematoxylin and bluing; original magnification X I25). (B)Horizontal PO section of whole globe OD "T" marks temporal side with subretinal and vitreous hemorrhage (H\&E, original scan magnification $X$ I); Inset: enlarged nasal retinal dehiscence near edge of disc (blue arrow); H\&E original magnification $X$ 31.25).[40 years in paraffin before APP-A4]

\section{Discussion}

The infarction of the optic nerve in Case \#2we believe expanded anteriorly from the CNS along its course to within $10 \mathrm{~mm}$ of the globe where it tapered anteriorly. Since the ON measures about $1 \mathrm{~mm}$ in the globe and $24 \mathrm{~mm}$ in the orbit the ON segment of approximately $29 \mathrm{~mm}$ would have extended into the chiasm. As additional evidence of chiasmal extension the posterior ON segment displayed dwindling pialseptae, characteristic of the chiasm. We suspect this injury was an extension of the global ischemic hypoxic injury (GHII) found during the neuropathology examination at autopsy. Normally, over the approximately $24 \mathrm{~mm}$ course from eye to chiasm, both internal and external carotid arterial input supply blood to the optic nerve. Compromise of the ophthalmic artery's orbital branches blood flows 
presumably occurs in GHII. To our knowledge, this case documents the first histological evidence of extension far anteriorly of GHII into the optic nerve. We otherwise found only suggestions and indirect evidence of such in experimental and human studies in current literature. Lamina cribrosa accumulation of APP-A4 after even transient IOP elevation would correlate well with past acute ocular hypertension experimental studies in foveated monkeys revealing lamina cribrosa blockade of both orthograde and retrograde axonal transport precisely in that location. ${ }^{15}$
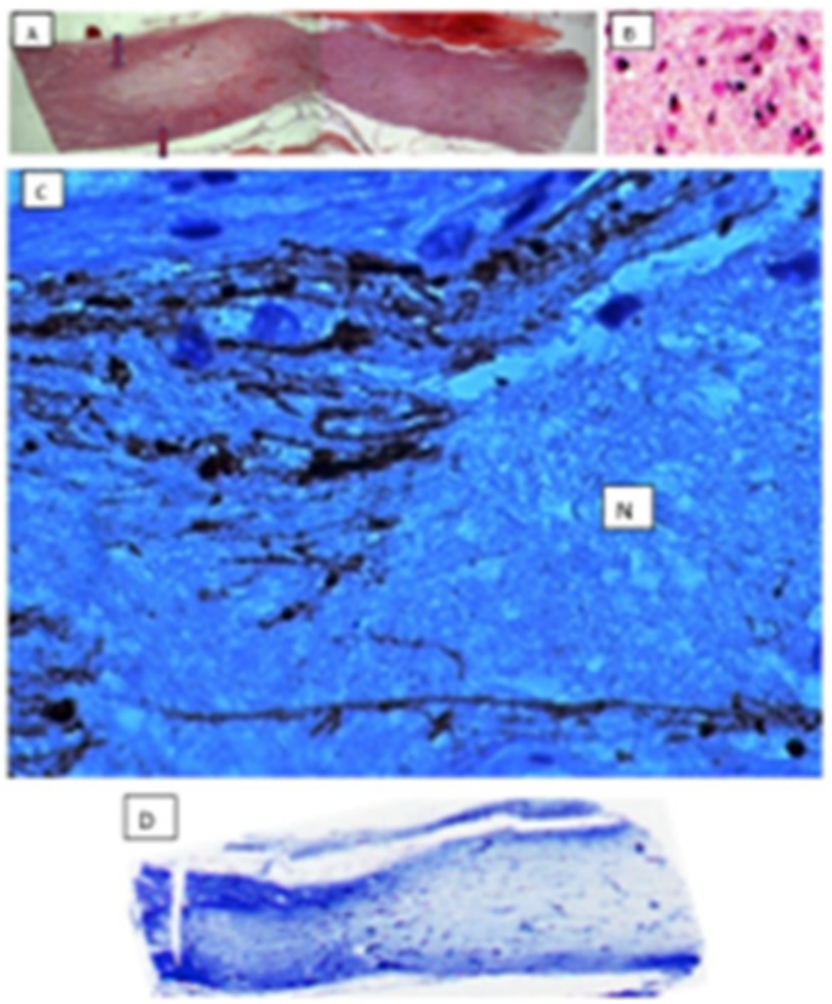

Figure 2 (A)Montage photomicrograph of longitudinal orbital optic nerve sample from Case \#2 OD, demonstrating infarct beginning about $1 \mathrm{~cm}$ posterior to the globe (pale wedge widening posteriorly from Eye-end [red arrows]. Subarachnoid hemorrhage marks CNS side where dwindling pialseptae identify the chiasm. Sharp edge on left side identifies Eye-end. The indentation mid-way is most likely a crush artifact acquired at tissue removal with parenchymal vascular congestion but no hemorrhage. (H\&E montage original magnification $X 31.25$ ). (B)Macrophages removing debris from infarct area near anterior end of lesion. (PAS; original magnification X 125). (C)Photomicrograph of APP-A4 accumulations (endbulb swellings) outlining infarct in above illustrations. The central part of the photo illustrates a large area of necrosis (N). (APP-A4 Hematoxylin and bluing; original magnification $X$ 500). (D)Photomicrograph Case \#2. Orbital optic nerve with eye end to left from deeper section demonstrating the enlarging pale area of necrosis and myelin loss expanding and extending nearly across the diameter of the posterior portion at the end of the approximately $29 \mathrm{~mm}$ optic nerve segment attached to the eye, by gross measure extending into the chiasm. This child's brain at autopsy demonstrated global hypoxic ischemic injury (GHII). (Betke $[K B]$ myelin stain enlarged from montage photomicrograph; original magnification $\times 31.25$ ).

\section{Conclusion}

Three of these four cases demonstrate previously unreported new findings related to optic nerve injury in AHT. Axonal end bulb swellings in the lamina cribrosa, as in Case \#4, have been previously reported using APP-A4 IHC without speculation as to the pathophysiology of their occurrence. ${ }^{8}$ We suspect that IOP is at least transiently elevated in fatal AHT cases probably due to several factors including high ICP and related migration of blood into the nerve's sheath spaces posterior to the globe, venous congestion of choroidal and retinal vessels, and vitreous hemorrhage in some with secondary hemorrhagic glaucoma. Ongoing studies of our whole series of AHT cases, to be reported in a separate manuscript, will explore correlations relevant to this concept.

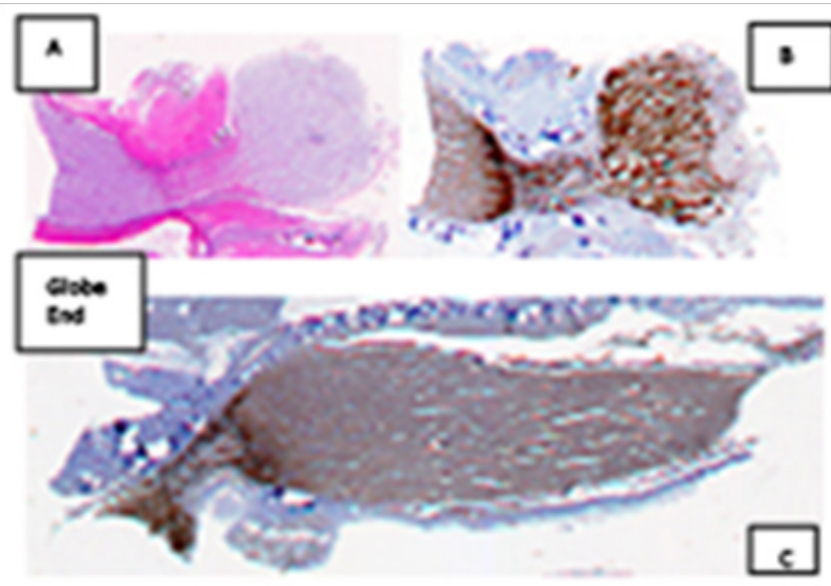

Figure 3 (A) Case \#3, Photomicrograph from asymmetrically longitudinally sectioned $24 \mathrm{~mm}$ orbital optic nerve segment OD.A cross section had been made $5 \mathrm{~mm}$ posterior to the sclera. A hemorrhagic parenchymal contusion approximately $6 \mathrm{~mm}$ posterior to the sclera is present surrounded by subdural blood (large blue arrow). (B) (APP-A4 Hematoxylin and bluingenlarged from IX scan, approximate magnification x 31.25). (B) (Photomicrograph H\&E enlarged from original magnification of 31.25).(C) Matching photomicrograph of adjacent gross specimen from opposite half of orbital nerve demonstrating heavy APP-A4 staining bracketing the same parenchymal injury in B. APP-A4 accumulations on the globe end of the contusion correlate with injury to rapid orthograde axonal transport. Accumulation of the marker on the CNS side of the injury implies response of retrograde axonal transport. (APP-A4 Hematoxylin and bluing; enlarged from Ix scan, magnification approximately $31.25)$.

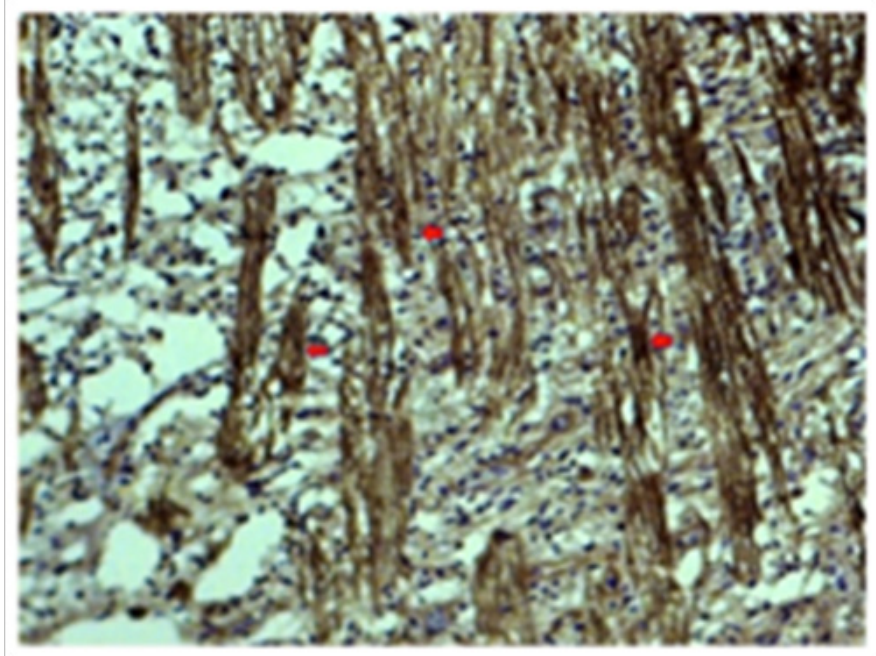

Figure 4 Case\#3 OS: Photomicrograph from temporal lamina cribrosa demonstrating what we interpret as multiple early forms of evolving endbulb swellings ( 3 red arrows) among many more in individual axons within midlaminar axon bundles which suggest axonal injury possibly secondary to transient elevation of IOP before death. (APP-A4 Hematoxylin and bluing; original magnification $\times 31.25$ ). 


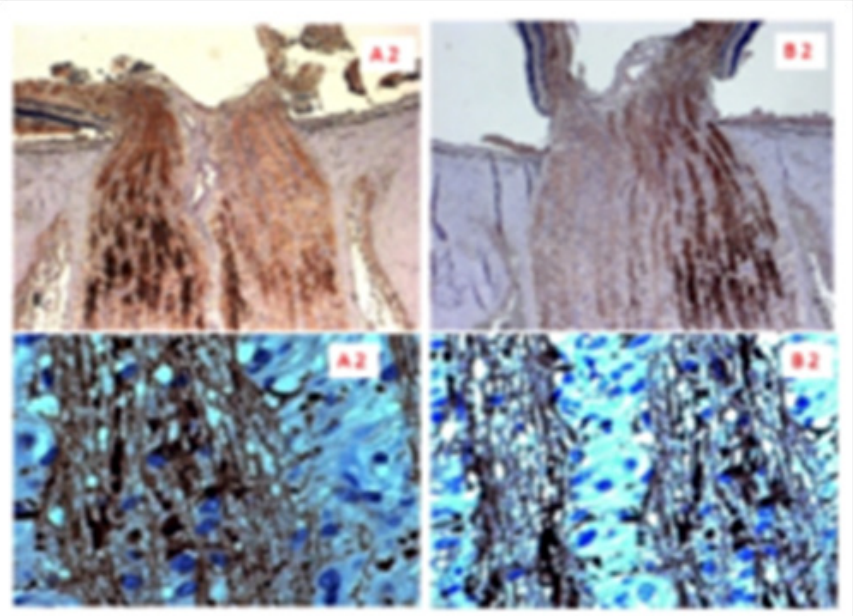

Figure 5 Case \#4: A2, B2Photomicrographs from OD (A2)and OS (B2) illustrating relatively symmetrical heavy macular APP-A4 input in horizontally oriented PO sections all APP-A4 Hematoxylin and bluing stained (Top: A2 \& B2 original magnification X 31.25; Bottom: A2 \& B2 well developed end-bulb swellings in lamina cribrosa axons OD and OS; original magnification $\times 500$ ).

Controversy persists among forensic pathologists and defense lawyers as to the validity of AHT diagnoses.1Even in instances where most of the typical findings of AHT are present, alternative diagnoses are often suggested by the defense and none of the typical findings are acknowledged as specific for AHT. ${ }^{3}$ Much research is yet needed to develop specific markers of AHT assuming they exist; neverthelessAPP-A4 can contribute valuable information to the diagnoses in these unfortunate and complex cases. ${ }^{7}$

\section{Acknowledgements}

Funding/support: Supported in part by an unrestricted grant from Research to Prevent Blindness.

\section{Conflict of interest disclosures}

Authors in this paper have no financial or proprietary interest in a product, method, or material published in this manuscript. All authors have completed and submitted the ICMUE Form for Disclosure of Potential Conflicts of Interest. All information and materials in the manuscript are original.

\section{IRB Approval}

University of California Irvine IRB approval not required for small case series with clinical-pathological correlation.

\section{Funding}

None.

\section{References}

1. Findley KA, Barnes PD, Moran DA, et al. Shaken Baby Syndrome, Abusive Head Trauma And Actual Innocence Getting It Right Houston. Journal of Health Law \& Policy Legal Studies Research Paper Series. 2012. p.209-312.

2. Bhardwaj G, Chowdhury V, Jacobs MB, et al. A Systematic Review of the Diagnostic Accuracy of Ocular Signs in Pediatric Abusive Head Trauma. Ophthalmology. 2010;117:983-992.

3. Levin AV. Retinal Hemorrhage in Abusive Head Trauma. Pediatrics online. 2010;2010-2020.

4. Emerson HV, Jakobs E, Green WR. Ocular Autopsy and Histopathologic Features of Child Abuse. Ophthalmology. 2007;114:1384-1394.

5. Matschke J, Püschel K, Glatzel M. Ocular pathology in shaken baby syndrome and other forms of infantile non-accidental head injury. IntJ Legal Med. 2009;123:183-197.

6. Ober RR. Hemorrhagic Retinopathy in Infancy A Clinicopathologic Report. JPediatrOphthalmol Strabismus. 1980;17(1):17-3.

7. Squire W. The Shaken Baby syndrome pathology and mechanisms. Acta Neuropathol. 2011;122(5):519-542.

8. Reichard RR, White CL, Hogan RN, et al. $\beta$-Amyloid Precursor Protein Immunohistochemistry in the Evaluation of Pediatric Traumatic Optic Nerve Injury. Ophthalmology. 2004;111(4):822-827.

9. Breazzanoa MP, Fang HS, Robinson MR, et al. AL Vitreomacular attachment ultrastructure and histopathological correlation. CurEye Res. 2016;41(8):1098-1104.

10. Dolinak D, Reichard R. Inflicted pediatric traumatic brain injury and $\beta$-APP IHC. CAP TODAY. 2006.

11. Johnson VE, Stewart W, Smith DH. Axonal Pathology in Traumatic Brain Injury. Exp Neurol. 2013;246:35-43.

12. Bais B, Kubat B, Motazedi E, et al. B-Amyloid Precursor Protein and Ubiquitin Immunohistochemistry Aid in the Evaluation of Infant Autopsy Eyes With Abusive Head Trauma. Am JOphthalmol . 2015;160(6):1285-1295.

13. IstvánMiklós, ZoltánZádori. Positive Evolutionary Selection of an HD Motif on Alzheimer Precursor Protein Orthologues Suggests a Functional Role. PLoS ComputBiolFeb. 2012;8(2):e1002356.

14. Lorenzo A, Deyts C, Kins S, et al. Axonal transport of APP and the spatial regulation of APP cleavage and function in neuronal cells. ExpBrain Res. 2012;217:353-364.

15. Minckler DS, Bunt AH and Johanson GW. Orthograde and retrograde axoplasmic transport during acute ocular hypertension in the monkey. Invest Ophthalmol Vis Sci . 1977;16(5):426-441. 